\title{
Un cas typique d'intoxication volontaire par l'éthylène glycol
}

\section{Typical case of self poisoning with ethylene glycol}

\section{Olivier LEMENAND(1), Françoise CANN ${ }^{(1)}$, Franck HERIAULT ${ }^{(2)}$, Robert LEROYER*(1)}

(1) Laboratoire de Pharmacie - Toxicologie, CHU Côte de Nacre - 14033 CAEN Cedex (2) Pharmacie du CHS "Bon Sauveur", 93, rue Caponière - 14000 CAEN

*Auteur à qui adresser la correspondance : Robert LEROYER, Pharmacie du CHU, Côte de Nacre 14033 CAEN Cedex - Tél : 0231064665 - Fax : 0231064667

(Reçu le 20 novembre 2000 ; accepté le 3 février 2000)

\section{$R E ́ S U M E ́$}

Le cas d'une intoxication volontaire aiguë par l'éthylène glycol, utilisé en tant que substitut à l'alcool chez un patient éthylique chronique, est rapporté.

Un suivi des taux plasmatiques d'éthylène glycol (EG) par une méthode enzymatique et de son principal métabolite, l'acide glycolique $(A G)$, par une méthode spectrophotométrique est présenté. Les concentrations maximales observées sont de 3,43 g/L pour l'EG et de $1,12 \mathrm{~g} / \mathrm{L}$ pour l'AG. La demi-vie d'élimination calculée de l'éthylène glycol $(7,4$ heures) est conforme au résultats de la littérature lors de la mise en auvre d'un traitement antidotique (fomépizole) et d'une épuration extra-rénale ; d'autre part, l'ampleur du trou anionique et le taux de glycolates sont superposables.

\section{MOTS-CLÉS}

Éthylène glycol, acide glycolique, intoxication.

\section{SUMMARY}

A typical case of self-poisoning with ethylene glycol used as a substitute to alcohol is reported.

The following of blood ethylen glycol and his main metabolite, glycolic acid, has been realized, the first one with an enzymatic assay, the other one with a spectrophotometric assay.

The highest observed values were : $3.43 \mathrm{~g} / \mathrm{L}$ for ethylene glycol and $1.12 \mathrm{~g} / \mathrm{L}$ for glycolic acid. Ethylene glycol elimination half life ( 7.4 hours), while the patient was treated by fomepizole and hemodialysis, is found similar to the reviews; furthermore, anion gap and glycolates rate were correlated.

\section{KEY-WORDS}

Éthylene glycol, glycolic acid, self-poisoning. 


\section{Introduction}

L'intoxication par l'éthylène glycol (EG) représente un danger vital par les troubles de l'homéostasie, du système nerveux central, du système cardio-vasculaire et de la fonction rénale que le métabolisme de ce composé est susceptible de provoquer $(1,2)$. En effet, l'éthylène glycol est oxydé par l'alcool déshydrogénase en composés acides dont les deux principaux sont l'acide glycolique (AG) et l'acide oxalique. Le premier est responsable en grande partie de l'acidose et du trou anionique rencontré dans cette intoxication (3), alors que le second est responsable d'une nécrose tubulaire rénale aiguë (4). Ces troubles peuvent être contrecarrés par la mise en route le plus précocement possible d'un traitement adéquat (5), d'où l'intérêt d'un diagnostic sûr et rapide qui, outre l'éthylène glycol, doit permettre d'identifier et de doser ses métabolites dans le sang et l'urine $(6,7)$.

Nous rapportons le cas d'une intoxication aiguë par consommation d'antigel contenant de l'éthylène glycol chez un sujet éthylique chronique déjà connu pour avoir absorbé d'autres substituts à l'alcool.

\section{Observation}

M. L. (49 ans) est admis au service des urgences du C.H.U. de Caen pour une intoxication par l'éthylène glycol après absorption d'antigel. Il a déjà été hospitalisé dans ce service à trois reprises dans les jours et les semaines précédents pour intoxication par de l'alcool à brûler (éthylèmie à $1,45 \mathrm{~g} / \mathrm{l}$ et méthanolémie à $0,23 \mathrm{~g} / \mathrm{L}$ ), par absorption d'acétone (acétonémie à $1,16 \mathrm{~g} / \mathrm{L})$ et de white spirit, ces épisodes succédant à deux hospitalisations pour éthylisme aigu $(2,9 \mathrm{~g} / \mathrm{L}$ et 2,8 g/L à l'admission).

Sur le plan biologique, ce patient présente à l'admission :

- une osmolalité sanguine calculée à $284 \mathrm{mOsm} / \mathrm{Kg}$ d'eau (trou osmolaire non mesuré)

- un trou anionique à $42,3 \mathrm{mmol} / \mathrm{L}(\mathrm{N}: 12$ à $16 \mathrm{mmol} / \mathrm{L})$

- un taux de bicarbonates sanguin effondré : $5 \mathrm{mmol} / \mathrm{L}$

$(\mathrm{N}: 22$ à $26 \mathrm{mmol} / \mathrm{L})$

- un pH artériel à 6,94 (N : 7,38 à 7,42)

- une $\mathrm{pCO}_{2}$ à $2,1 \mathrm{kiloPascal}(\mathrm{N}: 4,8$ à $5,6 \mathrm{kPa})$

Le dosage sanguin de l'éthylène glycol révèle une concentration de $2,25 \mathrm{~g} / \mathrm{L}$ et celui de l'acide glycolique une concentration de $1,12 \mathrm{~g} / \mathrm{L}$.

$\mathrm{Au}$ total, le patient présente donc un tableau d'intoxi- cation à l'éthylène glycol avec un trou anionique augmenté et une acidose métabolique franche. Par contre, l'osmolalité plasmatique n'ayant pas été mesurée, il est impossible d'évaluer l'ampleur du trou osmolaire, dont l'existence est cependant fortement probable.

Le patient est transféré dans le service de réanimation médicale où un traitement antidotique par 4-méthylpyrazole (fomépizole) et une épuration extra-rénale (hémodiafiltration) sont mis en route.

Des prélèvements artériels et veineux sọnt effectués régulièrement pour le suivi biochimique et toxicologique ; en particulier, des dosages de l'éthylène glycol et de l'acide glycolique sont réalisés toutes les 6 heures.

\section{Matériel et méthodes}

\section{Dosage de l'éthylène glycol}

Dans le laboratoire, nous utilisons une technique enzymatique de dosage de l'éthylène glycol dérivée de celles décrites par Mahly (9) et Goulle (10). Elle utilise la glycérol déshydrogénase et a été adaptée de sorte qu'elle soit réalisée sur un automate Cobas Mira Plus ${ }^{\circledR}$ (Roche Diagnostics). La réaction d'oxydation de l'éthylène glycol par l'enzyme forme du NAD réduit qui est dosé dans l'UV à $340 \mathrm{~nm}$.

Les réactifs chimiques utilisés sont de qualité pour analyses:

- solution de glycérol déshydrogénase provenant d'Enterobacter aerogenes (Boehringer, réf. 258555)

- éthylène glycol (Merck, réf. 9621): pour constituer une gamme étalon allant de 0 à $600 \mathrm{mg} / \mathrm{L}$ par surcharge de sérum de contrôle Seronorm (Fumouze ; France)

- solution de nicotinamide dinucléotide (NAD) (Boehringer, réf. 837067) dans du tampon tris 0,1 M (Prolabo, réf. 28.812.232)

\section{Dosage de l'acide glycolique}

La méthode spectrophotométrique que nous utilisons a été décrite par Fraser (11), il s'agit d'une mesure d'absorbance à $580 \mathrm{~nm}$ après traitement du plasma par l'acide chromotropique à chaud en milieu sulfurique concentré.

Les réactifs utilisés sont de qualité pour analyses : acide chromotropique (Prolabo, réf. 20.261.132) acide glycolique (Prolabo, réf. 20.336.131) - acide sulfurique concentré (Prolabo, réf. 20.700.298) - acide trichloracétique (Prolabo, réf. 20.734.295). 


\section{Demi-vie d'élimination}

Elle est déduite de la pente de la droite de régression du logarithme népérien de la concentration en fonction du temps, par la relation $\mathrm{t} 1 / 2=0,693 / \mathrm{k}_{\mathrm{e}}$.

\section{Résultats}

Les paramètres biochimiques mesurés de l'admission jusqu'à la soixante huitième heure sont indiqués dans le tableau I . Les valeurs de $\mathrm{pH}$ et $\mathrm{pCO} 2$ ont été déterminées $1 \mathrm{~h}$ et $6,5 \mathrm{~h}$ après l'admission; les valeurs sont respectivement de 6,87 et 7,14 pour le $\mathrm{pH}$ et 2,1 et 1,8 kiloPascals pour la $\mathrm{pCO} 2$.

On constate que la concentration de bicarbonates et le trou anionique se normalisent aux environs de la trente deuxième heure suivant l'admission aux urgences. Une hyperglycémie est à noter durant les vingt premières heures de l'intoxication, elle est imputable à un syndrome adrénergique.

Une recherche de cristaux d'oxalate de calcium dans les urines s'est révélée positive à la douzième heure suivant l'admission.
Nous avons mesuré les concentrations d'éthylène glycol et de glycolate toutes les 6 heures. Les résultats sont reportés dans le tableau II. Un pic d'éthylène glycol survient une heure après l'admission ce qui va dans le sens d'une ingestion récente de l'antigel confirmée par la présence d'éthylène glycol dans le liquide gastrique $(3.95 \mathrm{~g} / \mathrm{l})$. La concentration d'éthylène glycol décroît ensuite de manière exponentielle. Une représentation semi-logarithmique des données (figure 1) permet d'estimer la demi-vie d'élimination du toxique qui est ici de 7,4 heures $\left(k_{e}=0,094 h^{-1}\right)$.

La concentration d'acide glycolique décroît légèrement puis se maintient entre 0,90 et $1 \mathrm{~g} / \mathrm{L}$ pendant les 15 premières heures suivant l'admission, puis décroît de nouveau, mais cette fois-ci proportionnellement au temps.

Les concentrations des deux molécules deviennent inférieures à la limite de détection environ cinquante heures après l'admission tandis que les paramètres biochimiques sont déjà normalisés à ce moment (trou anionique à $13,5 \mathrm{mmol} / \mathrm{L}$ et bicarbonates à $30 \mathrm{mmol} / \mathrm{L})$.

Tableau I : Paramètres biochimiques (mmoleh) en fonction du temps.

\begin{tabular}{|c|c|c|c|c|c|c|c|c|}
\hline $\begin{array}{l}\text { Heures après admissions } \\
\text { Hed }\end{array}$ & 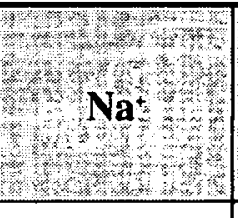 & (5) & $\begin{array}{l}\text { Glycémie } \\
\text { Ger }\end{array}$ & 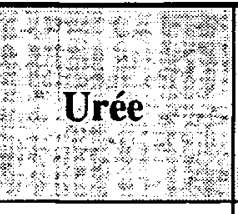 & 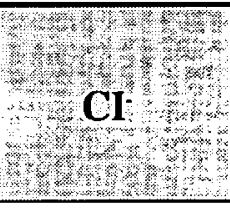 & HCO3 & $\begin{array}{l}\text { Osmolalité } \\
\text { (1) }\end{array}$ & $\begin{array}{l}\text { Trou } \\
\text { anionique } \\
\text { (2) }\end{array}$ \\
\hline 0 & 130 & 5,3 & 14,6 & 8,1 & 88 & 5 & 284,4 & 42,3 \\
\hline 6,5 & 141 & 4,2 & 20,7 & 10,3 & 83 & 4 & 315,3 & 58,2 \\
\hline 8 & 141 & 4,3 & 14,5 & 10 & 85 & 4 & 308,3 & 56,3 \\
\hline 12 & 139 & 4,2 & 17,6 & 9,4 & 90 & 7 & 307,0 & 46,2 \\
\hline 14,5 & 141 & 4,2 & 7,9 & 8,1 & 100 & 7 & 299,2 & 38,2 \\
\hline 20 & 139 & 4,6 & 9,2 & 7,0 & 97 & 13 & 295,4 & 33,6 \\
\hline 25,5 & 137 & 4,1 & 7,8 & 6,7 & 100 & 17 & 289,6 & 24,1 \\
\hline 32 & 138 & 3,8 & 8,2 & 6,8 & 100 & 22 & 292,1 & 19,8 \\
\hline 39,5 & 138 & 3,7 & 7,0 & 6,9 & 100 & 28 & 290,9 & 13,7 \\
\hline 50 & 139 & 3,5 & 6,0 & 7,7 & 98 & 31 & 292,7 & 13,5 \\
\hline 55,5 & 136 & 3,4 & 6,9 & 9,9 & 100 & 26 & 290,1 & 13,4 \\
\hline 68 & 135 & 3,5 & 6,3 & 15,1 & 99 & 25 & 293,0 & 14,5 \\
\hline Normales du laboratoire & $135-145$ & $3,6-4,8$ & 3,6 à 5,5 & 2,5 à 6 & 95 à105 & 24 à 30 & $270-290$ & $12-16$ \\
\hline
\end{tabular}

(1) Osmolalité calculée $=\left(1,8 . \mathrm{Na}^{+}+\right.$Urée + Glycémie $) / 0,93-\left(\mathrm{mOsM} / \mathrm{Kg} \mathrm{H}_{2} \mathrm{O}\right)$

(2) Trou Anionique $=\left(\mathrm{Na}^{+}+\mathrm{K}^{+}\right)-\left(\mathrm{Cl}+\mathrm{HCO}_{3}{ }^{-}\right)$ 
Tableau II : Évolution de l'éthylène-glycol et de l'acide glycolique en fonction du temps.

\begin{tabular}{|c|c|c|}
\hline $\begin{array}{c}\text { Heures } \\
\text { après } \\
\text { admission }\end{array}$ & $\begin{array}{c}\text { Éthylène } \\
\text { glycol } \\
\text { (g/l) }\end{array}$ & $\begin{array}{c}\text { Acide } \\
\text { glycolique } \\
\text { (g/) })\end{array}$ \\
\hline 0 & 2,25 & 1,12 \\
\hline 1 & 3,43 & 0,77 \\
\hline 7,50 & 3,13 & 0,94 \\
\hline 13,5 & 1,36 & 0,89 \\
\hline 19,5 & 0,86 & 0,66 \\
\hline 25,5 & 0,54 & 0,47 \\
\hline 31,5 & 0,34 & 0,33 \\
\hline 37,5 & 0,17 & 0,19 \\
\hline 43,5 & 0,13 & 0,10 \\
\hline 49,5 & 0,04 & 0,04 \\
\hline 55,5 & ND & ND \\
\hline 61,5 & ND & ND \\
\hline
\end{tabular}

\section{Discussion}

L'utilisation d'une méthode enzymatique simple à mettre en œuvre pour le dosage de l'EG permet $\mathrm{d}$ 'orienter très rapidement le diagnostic en cas de résultat positif $(10,12)$; cependant, une réponse négative n'exclut pas, en cas d'acidose associée à un trou anionique et un trou osmolaire, une intoxication par EG détectée tardivement (13) en raison de sa courte demivie (3-8 h sans traitement). La disponibilité d'une méthode colorimétrique, facile à réaliser et de suffisamment bonne spécificité $(11,14)$ est intéressante. En effet, les méthodes chromatographiques de dosage simultané ou non de EG et AG $(11,15)$ ne sont pas accessibles à tous les laboratoires et leur utilisation en urgence est délicate, vu leur durée et leur complexité.

Le cas rapporté est très représentatif des intoxications aiguës volontaires. L'acidose marquée et l'effondrement des bicarbonates sont les principaux signes biochimiques observés avec un couple $\mathrm{pH}=6,87 /$ bicarbonates $=4,0 \mathrm{mmol} / \mathrm{L}$, à rapprocher de la revue de 79 observations en 12 ans très récemment publiée (16) dont un couple record à $6,43 / 1,36 \mathrm{mmol} / \mathrm{l}$ (17). Il faut noter l'importance du trou anionique qui va atteindre $58 \mathrm{mmol} / \mathrm{L}$ et son évolution très superposable à celle de l'AG (figure 2). Une acidose très modérée voire absen-

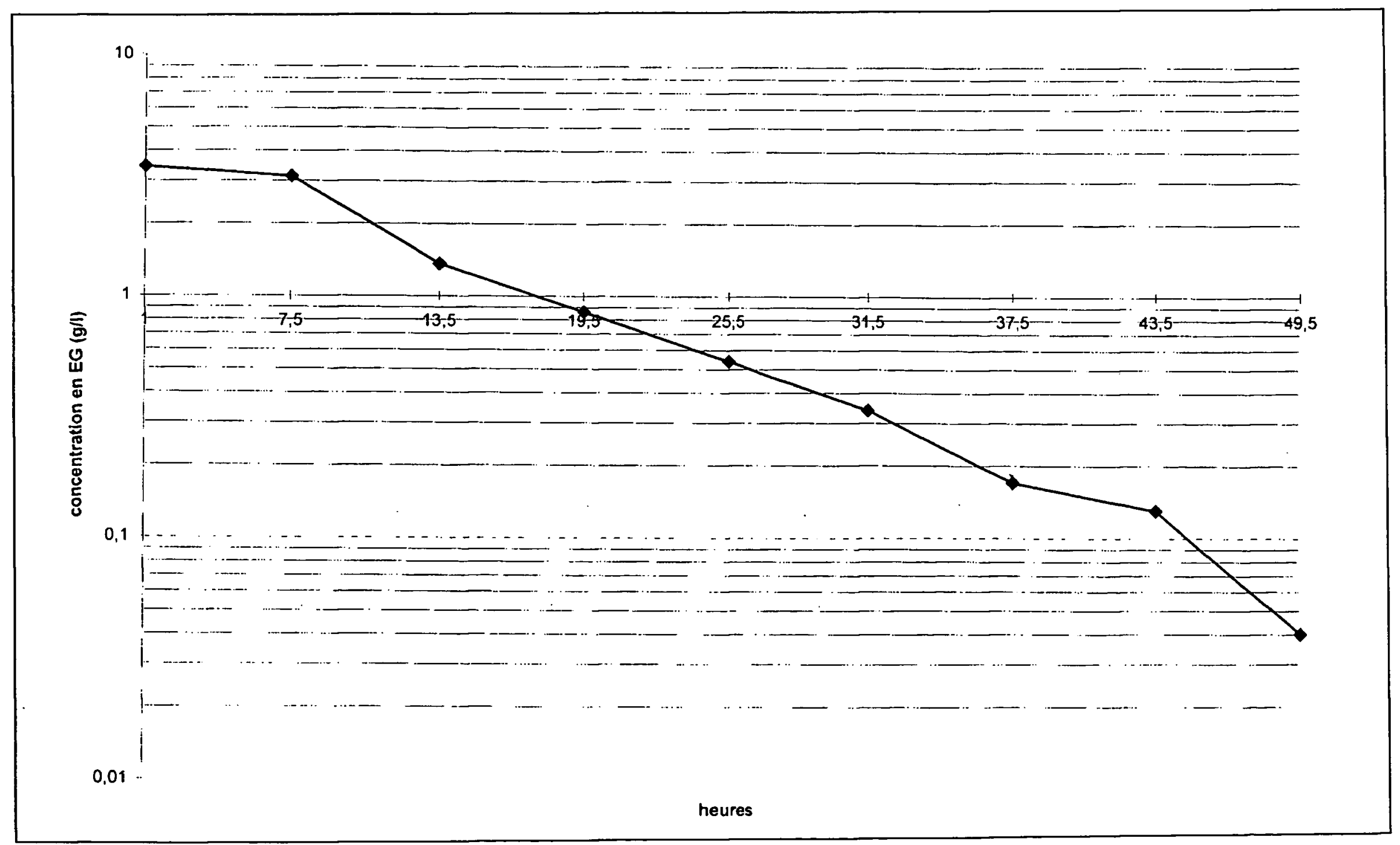

Figure 1 : Évolution des concentrations en EG (coordonnées semi-log.) 
te au regard de l'absorption d'une quantité d'EG peut s'observer dans quelques cas où une absorption préalable ou simultanée d'alcool a un effet antidotique $(18,19,20)$; dans ce cas le métabolisme en AG est en partie inhibé.

L'osmolalité calculée, qui ne tient pas compte des métabolites de l'éthylène glycol, est élevée surtout de part l'hyperglycémie pendant les premières heures; les concentrations en EG et AG vont également l'accroître de 50 à 60 mOsm bien que la mesure n'ait pas été effectuée, le trou osmolaire pouvant atteindre jusqu'à 170 mOsM (21).

Si le dosage de l'EG est loin des records publiés : 8,88 $\mathrm{g} / \mathrm{l}(19)$ et $7,91 \mathrm{~g} / \mathrm{l}(21)$, sa détermination toutes les 6 heures a permis de calculer sur 9 points une demi-vie de 7,4 heures, augmentée par rapport à la demi-vie sans traitement de la molécule. Cette demi-vie est modifiée par deux phénomènes contraires : l'administration de fomépizole qui tend à l'augmenter d'une part (15-25 heures) (22) et l'épuration extra-rénale qui tend à la diminuer d'autre part (2,5-3,5heures) (7). La décroissance de l'AG suit une cinétique d'ordre zéro et ne permet pas de calculer de $\mathrm{T} 1 / 2$ mais son élimination est lente en accord avec différentes observations $(6,7)$; de même, la corrélation avec le trou anionique est bien vérifiée (7).

\section{Conclusion}

Nous avons mesuré l'éthylène glycolémie et la glycolatémie chez un patient ayant consommé de l'antigel en tant que substitut alcoolique. La demi-vie de l'EG observée est de l'ordre de celle attendue chez un patient dialysé et traité par fomépizole. Le trou anionique est bien corrélé au taux de glycolates.

Le patient s'est remis de cet épisode sans séquelle malgré des perturbations assez importantes des paramètres biochimiques et ce sans doute grâce à une prise en charge rapide de l'intoxication et une mise en route sans tarder du traitement antidotique (4-méthylpyrazole et épuration extra-rénale). Cette observation confirme s'il en était besoin, l'importance du facteur temps dans ce type d'intoxication.

Epilogue : Après un séjour en service de psychiatrie succédant à cet épisode d'intoxication, le patient a de nouveau été admis aux urgences du C.H.U de CAEN pour deux nouveaux épisodes d'intoxication aiguë (éthylèmie à $3,41 \mathrm{~g} / \mathrm{l}$ puis acétonèmie à $2,18 \mathrm{~g} / \mathrm{l}$ ).

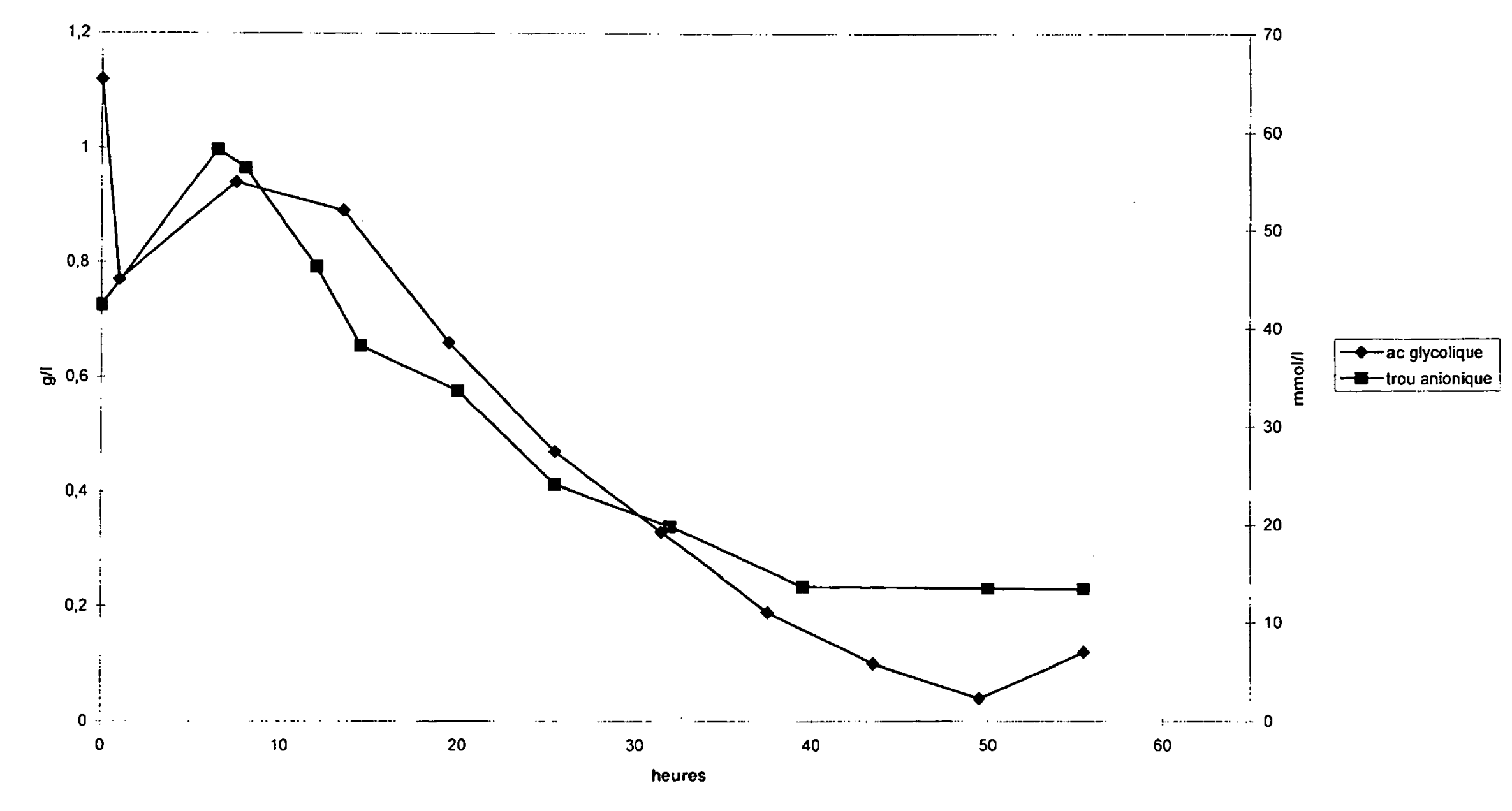

Figure 2 : Relation acide glycolique/trou aniomique. 


\section{Références}

1. Pincus K.T., Shinn B.W., Frock J.T. Ethylene glycol poisoning revisited -Hosp.Pharm. 1996 ; 31 : 536-40.

2. Gaulier J.M., Gillet Y. , Lardet G., Cochat P. Intoxication par l'éthylène glycol - Lyon Pharm. 1997 ; 48 : 370-2.

3. Adrogue H.J., Madias N.E. Management of life-threatening acid-base disorders -N.Engl.J.Med. $1998 ; 338(1)$ : 26-34.

4. Jacobsen D.,Hewlett T.P., Webb R. Brown S.T., Ordinario A.T., Mc Martin Ethylene glycol intoxication: evaluation of kinetics and crystalluria - Am.J.Med. $1988 ; 84: 145-52$.

5. Gaillard Y., Auboyer C., Guy C., Gay-Montchamp J.P., Ollagnier M. Schéma d'un arbre décisionnel à propos d'une intoxication aiguë par l'éthylène glycol. - Lyon Pharm. 1993 ; 44 : 259-63.

6. Hewlett T.P., Mc Martin K.E., Lauro A.J., Ragan F.A. Ethylene glycol poisoning.The value of glycolic acid determinations for diagnosis and treatment Clin.Toxicol. 1986 ; 24(5) : 389-402.

7. Moreau C.L., Kerns W., Tomaszewski C.A., Mc Martin K.E., Rose R., Ford M.D., Brent J., and the META study group. Glycolate kinetics and hemodialysis clearance in ethylene glycol poisoning. Clin.Toxicol. 1998 ; 36(7) : 659-66.

8. Jacobsen D., Ovrebo S., Ostborg J., Sejersted O.M. Glycolate causes the acidosis in ethylene glycol poisoning and is effectively removed by hemodialysis - Acta Med.Scand. 1984 ; 216 :409-16.

9. Mahly M., Lardet G., Vallon J.J. Automated Cobas Mira kinetic enzymatic assay for ethylene glycol applied to emergency situations - J.Anal.Toxicol. 1994 ; 18 : 269-71

10. Goulle J.P., Allion M.J. Anagnostides J.G. Ethylène glycol : intérêt d'une méthode enzymatique en toxicologie d'urgence - Toxicorama 1995 ; VII : 15-20.

11. Fraser A.D., Mc Neil W. Colorimetric and gas chromatographic procedures for glycolic acid in serum : the major toxic metabolite of ethylene glycol - Clin.Toxicol. 1993; $31(3): 397-405$.
12. Malandain H., Cano Y. Des méthodes enzymatiques simples, utilisables en garde, pour doser le méthanol, l'éthylène glycol et l'acide glycolique - Toxicorama 1995 ; VII : 31-7.

13. Fraser A.D. Ethylene glycol, glycolic acid, and metabolic acidosis of unknown origin - Clin.Chem. 1993 ; 39(8) : 1753-54.

14. Fraser A.D. Importance of glycolic acid analysis in ethylene glycol poisoning - Clin.Chem. 1998 ; 44(8) : 1769.

15. Yao H.H., Porter W.H. Simultaneous determination of ethylene glycol and its major toxic metabolite, glycolic acid, in serum by gas chromatography. Clin.Chem. 1996 ; 42 (2) : 292-97.

16. Wisse B., Thakur S., Baran D. Recovery from prolonged metabolic acidosis due to accidental ethylene glycol poisoning - Am.J.Kidney Dis. 1999 ; 33(2) : E4.

17.Blakeley K.R., Rinner S.E., Knochel J.P. Survival of ethylene glycol poisoning with profound acidemia N.Engl.J.Med. 1993 ; 328(7) : 515-16

18. Divanon F., Leroyer R., Leprince M.C., Riby J.P., Collet $\mathrm{C}$. A propos d'une intoxication par l'éthylène glycol - J.Pharm.Clin. 1997 ; $16: 177-82$.

19.Davis D.P., Bramwell K.J., Hamilton R.S., Williams S.R. Ethylene glycol poisoning : case report of a record-high level and a review - J.Emerg.Med. 1997 ; 15(5) : 653-67.

20. Ammar K.A., Heckerling P.S. Ethylene glycol poisoning with a normal anion gap caused by concurrent ethanol ingestion : importance of the osmolal gap - Am.J.Kidney Dis. $1996 ; 27: 130-3$.

21.Eder A.F., McGrath C.M., Dowdy Y.G., Tomaszewski J.E., Rosenberg F.M., Wilson R.B., Wolf B.A., Shaw L.M. Ethylene glycol poisoning : toxicokinetic and analytical factors affecting laboratory diagnosis Clin.Chem. 1998 ; 44(1) : 168-77.

22. Brent J., McMartin K., Phillips S., Burkhart K.K., Donovan J.W., Wells M., Kulig K. Fomepizole for the treatment of ethylene glycol poisoning - N.Engl.J.Med $1999 ; 340(11): 832-8$. 